\title{
Update on Targeted Therapies for Advanced Non-Small Cell Lung Cancer: Durvalumab in Context
}

This article was published in the following Dove Press journal:

OncoTargets and Therapy

\begin{abstract}
Sneha Gullapalli'
Jordi Remon (D) ${ }^{2}$

Lizza EL Hendriks ${ }^{3}$

Gilberto Lopes ${ }^{4}$

'Division of Pulmonary and Critical Care, University of Miami, Jackson Memorial Hospital, Miami, FL, USA; ${ }^{2}$ Department of Medical Oncology, Centro Integral Oncológico Clara Campal (HM-ClOCC), Hospital HM Delfos, HM Hospitales, Barcelona, Spain; ${ }^{3}$ Department of Pulmonary Diseases GROW - School for Oncology and Developmental Biology, Maastricht University Medical Center+, Maastricht, the Netherlands; ${ }^{4}$ Divisions of Hematology and Medical Oncology, Departments of Medicine, Miller School of Medicine, University of Miami, Sylvester Comprehensive Cancer Center, Miami, FL, USA
\end{abstract}

\begin{abstract}
Immune checkpoint inhibitors (ICIs) have transformed the therapeutic strategy and prognosis of advanced non-small cell lung cancer (NSCLC) patients. Nowadays, ICIs as monotherapy or in combination with chemotherapy are the standard of care treatment in advanced NSCLC, and in stage III, durvalumab (a programmed death ligand 1 inhibitor) is the unique drug approved as consolidation treatment after chemo-radiotherapy. This article reviews the pharmacological properties, clinical activity and safety of durvalumab as monotherapy or in combination with chemotherapy or other ICIs in the therapeutic strategy of NSCLC patients.
\end{abstract}

Keywords: durvalumab, non-small cell lung cancer, PACIFIC, rechallenge, MYSTIC

\section{Introduction}

In recent years, two major improvements in the treatment of patients with advanced non-small cell lung cancer (NSCLC) have taken place. The first is genomic profiling with the identification of oncogenic addicted tumors allowing for personalised treatment, mainly in adenocarcinomas. The second is the introduction of immune checkpoint inhibitors (ICI). These treatments have dramatically changed the treatment paradigm and prognosis of patients with NSCLC. Several ICIs have been tested in NSCLC, such as inhibitors of cytotoxic T lymphocyte-associated antigen 4 (CTLA-4) like tremelimumab and ipilimumab, as well as programmed cell death protein (ligand) 1 (PD-[L])1) inhibitors such as nivolumab, pembrolizumab (both PD-1 inhibitors) and atezolizumab, durvalumab and avelumab (PD-L1 inhibitors). This review provides an overview of the preclinical and clinical efficacy of durvalumab in the treatment of NSCLC.

\section{Overview of Treatment Strategies with ICI in advanced Setting}

In the first-line setting, ICIs have become standard of care (SoC) either as monotherapy (pembrolizumab for tumours with high programmed cell death ligand-1 (PD-L1) expression ( $\geq 50 \%$ ) in Europe or with PD-L1 $\geq 1 \%$ in the United States of America), ${ }^{1,2}$ regardless of histologic subtype, or in combination with chemotherapy regardless of PD-L1 expression in non-squamous (pembrolizumab/atezolizumab plus platinum-doublet chemotherapy, or atezolizumab-carboplatin-paclitaxelbevacizumab) ${ }^{3-7}$ and squamous histology (pembrolizumab plus paclitaxel and carboplatin). ${ }^{8}$ The introduction of ICIs in the first-line treatment of metastatic NSCLC has marked a step forward in the therapeutic strategy of this patient
Correspondence: Jordi Remon Department of Medical Oncology, Centro Integral Oncológico Clara Campal (HM-

CIOCC), Hospital HM Delfos, HM

Hospitales, Avinguda Vallcarca I5I,

Barcelona 08023, Spain

Tel +34932545029

Email jordi.remon@delfos.cat 
population, improving both the outcome and quality of life compared with platinum-based chemotherapy. ${ }^{9-11}$ Although the upfront combination of nivolumab plus ipilimumab has reported survival benefit compared with standard platinum-based chemotherapy in PD-L1 $\geq 1 \%$ tumours, ${ }^{12}$ it is not yet approved by health authorities and EMA application for this combination was withdrawn.

In platinum-refractory advanced NSCLC patients not treated with ICI in the first-line setting, three anti-PD(L)1 drugs are $\mathrm{SoC}$ in second-line. These are pembrolizumab in PD-L1 positive tumours and nivolumab and atezolizumab regardless of PD-L1 expression based on the survival benefit compared with standard docetaxel. ${ }^{13-16}$

Compared with historical data, the introduction of ICIs in the therapeutic strategy of advanced NSCLC patients markedly improved the 5-year overall survival (OS). Based on the phase 1b KEYNOTE 001 trial, monotherapy pembrolizumab resulted in a 5 -year OS of $29.6 \%$ and $15.7 \%$ in treatment-naïve patients with a PD-L1 of $\geq 50 \%$ and $1-49 \%$, respectively. For pretreated patients, 5-year OS was $25.0 \%$ and $12.6 \%$, respectively. On the contrary, for those with a PD-L1 of $<1 \%$, survival was only $3.5 \%$ and resembles the survival in the pre-ICI era. ${ }^{17}$ For nivolumab, 5-year OS was $16 \%$ reaching $43 \%$ in the small subgroup of PD-L1 $\geq 50 \%$. ${ }^{18}$ This long-term survival benefit with ICI has been confirmed in Phase III clinical trials in pre-treated NSCLC patients either with pembrolizumab (KEYNOTE 010,3 -year OS of $23 \%$ in PD-L1 $\geq 1 \%$ ) or nivolumab (pooled 5-year OS from CheckMate 017 and 057 trials of $13.4 \%) .{ }^{19}$ For the combination of chemotherapy plus ICI, 5-year OS data are not available. The longest follow-up comes from the KEYNOTE 189 trial (platinumpemetrexed-pembrolizumab), which reported after a median follow-up of 23.1 months, 2-year OS of $51.9 \%$, $44.3 \%$ and $38.5 \%$ for PD-L1 $\geq 50 \%$, PD-L1 $1-49 \%$ and PD-L1 $<1 \%$, respectively. ${ }^{20}$

\section{How Does Durvalumab Fit in the Treatment Regimen of NSCLC? Durvalumab}

Programmed cell death ligand 1 (PD-L1) binds to two regulatory receptors on T cells: PD-1 and CD80 (B7.1). Binding of PD-L1 to PD-1 inhibits T-cell proliferation and binding of PD-L1 to CD80 blocks T-cell activation, hindering antitumor responses. Durvalumab (Imfinzi, MEDI4736) is a selective, high-affinity human immunoglobulin G1 (IgG1) monoclonal antibody that inhibits PD-
L1 binding to programmed death 1 (concentration that inhibits 50\%[IC50]: $0.1 \mathrm{nM}$ ) and CD80 (IC50: $0.04 \mathrm{nM}$ ). It is highly selective for PD-L1 and does not bind to PDL2. ${ }^{21}$ IgG1 isotype antibodies may trigger cytotoxic effects, such as cell-mediated cytotoxicity via antibodies or complement. An engineered triple mutation of IgG1 heavy chain in the $\mathrm{FC}$ region antibody domain is designed to avert the activity related to antibody-dependent cellmediated cytotoxicity and complement-dependent cytotoxicity. ${ }^{22}$ The pharmacokinetics (PK) of durvalumab was studied at doses ranging from 0.1 to $20 \mathrm{mg} / \mathrm{kg}$ administered every 2, 3, or 4 weeks. The PK exposure of durvalumab increases more than dose proportionally at doses of $<3 \mathrm{mg} / \mathrm{kg}$ and dose proportionally at doses of $\geq 3 \mathrm{mg} / \mathrm{kg}$, with the steady state achieved at around 16 weeks. Durvalumab clearance decreases over time, with a terminal half-life of approximately 17 days. ${ }^{22}$ According to PK data, it does not seem necessary to adjust dose for durvalumab, as PK is not clinically affected by age (19-96 years), body weight (34-149 kg), sex, albumin levels, tumor type, race, mild and moderate renal impairment (creatinine clearance $60-89$ and $30-59 \mathrm{~mL} / \mathrm{min}$ ), and mild hepatic impairment (bilirubin $\leq$ upper limit of normal [ULN] and aspartate aminotransferase (AST) $>$ ULN or bilirubin $>1.0-1.5 \times$ the ULN and any AST). However, it is unknown whether severe renal impairment or moderate or severe hepatic impairment influences the PK of durvalumab. $^{22}$

\section{Durvalumab in Previously Treated Patients}

The phase I/II 1108 study evaluated the safety and clinical activity of durvalumab in several tumor types. In the doseescalation phase, the dose of $10 \mathrm{mg} / \mathrm{kg}$ was chosen to ensure a mean trough concentration of $50 \mathrm{mcg} / \mathrm{mL}$ and no dose-limiting toxicities were observed at that dose. In the expansion phase, 304 stage IIIB-IV NSCLC patients were included testing durvalumab at $10 \mathrm{mg} / \mathrm{kg}$ every 2 weeks (Q2W) for 12 months or until progressive disease (PD). Initially, patients were enrolled regardless of tumor PD-L1 expression, but a protocol amendment required that all remaining non-squamous patients had positive PD-L1 expression ( $\geq 25 \%$ by Ventana SP263 assay). Of note, $21.1 \%$ of patients were treatment-naïve and received durvalumab in the first-line setting, and 165 had PD-L1 expression $\geq 25 \%$. The RRs were $26 \%$ and $12.7 \%$ in firstline and previously treated patients, respectively, without 
differences according to histological subtype (14.0\% in squamous and $16.7 \%$ in non-squamous). Median progression-free survival (PFS) and OS were 1.7 months and 12.4 months, respectively. According to PD-L1 expression, the RR (21.8\% versus $6.4 \%$ ), PFS (2.6 versus 1.4 months) and OS (16.4 versus 7.6 months) appeared to be enhanced in patients with greater PD-L1 expression $(\geq 25 \%$ versus $<25 \%$, respectively). In $\mathrm{PD}-\mathrm{L} 1 \geq 25 \%$ and treatmentnaïve patients durvalumab reported a RR, PFS and OS of 26.8\%, 5.4 months and 21.9 months, respectively. Treatment-related adverse events (TRAE's) occurred in $57.2 \%$, being grade $\geq 3$ in $10.2 \%$ of patients, and led to discontinuation in $5.6 \%$ of patients enrolled. No differences in any $\mathrm{AE}$ or grade $\geq 3$ AEs were reported according to PD-L1 expression. ${ }^{23}$ Clinical activity of durvalumab in first- and second-line is consistent with data reported with other anti-PD(L)1 inhibitors.

The Phase II ATLANTIC trial is a single-arm, threecohort study that evaluated durvalumab $(10 \mathrm{mg} / \mathrm{kg}$ Q2W) as third-line or beyond in stage IIIB-IV NSCLC patients. Cohort 1 included $E G F R / A L K$-positive tumors $(\mathrm{N}=111)$, cohort $2 E G F R / A L K$ wild type $(\mathrm{N}=265)$, and cohort 3 $E G F R / A L K$ wild-type tumors with $\mathrm{PD}-\mathrm{L} 1$ expression $\geq 90 \% \quad(\mathrm{~N}=68)$. The primary endpoint was the $\mathrm{RR}$ in patients with increased tumour expression of PD-L1 (defined as $\geq 25 \%$ of tumour cells in cohorts 1 and 2 , and $\geq 90 \%$ of tumour cells in cohort 3 ), being $12.2 \%, 16.4 \%$ and $30.9 \%$, respectively. In cohort 1 and 2 , the RR was higher in tumors with PD-L1 $\geq 25 \%$ than in tumors with PD-L $1<25 \%$. Median PFS was 1.9 months, regardless of PD-L1 expression $(\geq 25 \%$ or $<25 \%$ ), 3.3 months ( $v s 1.9$ months in tumors with PD-L1 $<25 \%$ ) and 2.4 months. The 1 -year OS was $40 \%$ (vs $54.8 \%$ in tumors with PD-L1 $<25 \%$ ), $34.5 \%$ ( vs $47.7 \%$ in tumors with PD-L1 <25\%) and $50.8 \%$, respectively. Grade 3 or 4 TRAEs occurred in $40(9 \%)$ of 444 patients overall and serious TRAEs occurred in $27(6 \%)$ overall, with the most common pneumonitis, fatigue and infusion-related reactions with an incidence of $1 \%$ each, and $2 \%$ discontinued durvalumab due to TRAEs. ${ }^{24}$

Finally, in previously treated ( $\geq 2$ prior systemic treatments) advanced NSCLC patients, the phase III ARCTIC trial (NCT02352948) ${ }^{25}$ evaluated durvalumab $(10 \mathrm{mg} / \mathrm{kg}$ Q2W up to 12 months) versus SoC (erlotinib $150 \mathrm{mg}$, gemcitabine $1000 \mathrm{mg} / \mathrm{m}^{2}$ IV day 1,8 , and 15 of a 28day cycle; or vinorelbine $30 \mathrm{mg} / \mathrm{m}^{2}$ iv, day $1,8,15$, and 22 of a 28-day cycle) in 126 patients with PD-L1 $\geq 25 \%$ (substudy A). The combination of durvalumab plus tremelimumab (durvalumab $20 \mathrm{mg} / \mathrm{kg}$ IV + tremelimumab $1 \mathrm{mg} / \mathrm{kg}$ IV Q4W for up to 12 weeks then durvalumab 10 $\mathrm{mg} / \mathrm{kg}$ IV Q2W for 34 weeks) or either agent as monotherapy versus SoC in 469 patients with PD-L1 expression $<25 \%$ was evaluated in sub-study B. In sub-study A, the median OS was 11.7 and 6.8 months for durvalumab and SoC arm, respectively (HR 0.63 [0.42, 0.93]), with 1-year OS rates of $49.3 \%$ and $31.3 \%$, respectively. Median PFS was 3.8 and 2.2 months (HR 0.71 [0.49, 1.04]). Although durvalumab reduced the risk of death compared with chemotherapy, the study was underpowered to detect statistical significance. In sub-study B, the median OS was 11.5 versus 8.7 months with durvalumab plus tremelimumab versus SoC (HR 0.80 [95\% CI 0.61, 1.05]; p= 0.109 ), with 1 -year OS of $49.5 \%$ and $38.8 \%$, respectively. Median PFS was 3.5 months in both groups (HR 0.77 $[0.59,1.01] ; \mathrm{p}=0.056) .{ }^{25}$ Durvalumab plus tremelimumab did not meet the primary endpoints of statistically significant and clinically meaningful improvements in PFS and OS compared with SoC.

Finally, durvalumab has also been assessed in previously treated squamous advanced NSCLC patients in the phase II umbrella S1400A LUNG-Map Trial (NCT02766335). In this cohort, 68 patients received durvalumab and 30 docetaxel, $25 \%$ had PD-L1 $\geq 25 \%$. RR was $16.2 \%$ (14.3\% in PD-L1 $\geq 25 \%$ and $6.9 \%$ in PD-L1 $<25 \%$ ) versus $6.7 \%$ with docetaxel. Although there were no differences in the median PFS between durvalumab and docetaxel (2.8 and 2.9 months, respectively), PFS rate at 6 months was higher for durvalumab than docetaxel $(25 \%$ and $13.3 \%$, respectively). Median OS was 11.7 and 7.6 months, respectively, and grade $\geq 3$ TRAEs were reported in $34 \%$ of patients. ${ }^{26}$ These data confirm that ICI is the standard treatment as second-line advanced NSCLC patients not previously treated with ICI and who progress on platinum-based-chemotherapy. However, currently most patients receive ICI either as monotherapy or in combination with chemotherapy in the first-line setting, as it is the standard of care treatment. ${ }^{27}$ Therefore, the role of durvalumab in third line or beyond is very limited and not approved by health authorities. Of note, the knowledge of mechanisms of primary or acquired resistance on ICI is a challenge, and the role of ICI beyond progression, along with personalised treatment based on the mechanism of resistance is another challenge, being explored in the ongoing HUDSON trial (NCT03334617). 


\section{Durvalumab Monotherapy or in Combination in First-Line Setting}

PD-L1 expression is a predictive biomarker for ICI efficacy, and this benefit increases with higher PD-L1 expression. ${ }^{28}$ Data from the randomized phase III clinical trials such as KEYNOTE 024, KEYNOTE 042 and IMpower $110,^{2,29,30}$ have reported survival benefit with anti-PD(L)-1 drugs compared with platinum-based chemotherapy in PD-L1 positive tumors; ${ }^{2}$ achieving the maximum benefit among those tumors with higher PD-L1 expression. $^{2,29,30}$

The phase III PEARL trial (NCT03003962) assesses the outcome (PFS and OS) of first-line durvalumab $(20 \mathrm{mg} / \mathrm{kg}$ i. v. Q4W) compared with platinum-based chemotherapy in stage IV, EGFR/ALK wild-type NSCLC patients with high PD-L1 expression ( $\geq 25 \%$ tumour cells with membrane staining using the Ventana PD-L1 [SP263] Assay). Likewise, in advanced NSCLC patients, the efficacy of durvalumab in first-line setting or as a maintenance treatment after platinum-based chemotherapy has been explored in two phase II trials, the National Lung Matrix Trial (NCT02664935) and in the SAPHIR2-lung trial (IFCT1301), respectively, but the results have not been presented already.

In first-line setting, ICI-chemotherapy is SoC in advanced NSCLC patients whose tumors harbour a PD-L1 expression $\leq 50 \% .^{3,5,8}$ Recently, the phase III CheckMate 227 trial reported a significantly longer OS with the combination of nivolumab plus ipilimumab in the first-line setting compared with platinum-based chemotherapy in PD-L1 $\geq 1 \%$ NSCLC patients (17.1 months vs 14.9 months, $\mathrm{p}=0.007$ ). However, this benefit occurred regardless of PDL1 or tumor mutational burden (TMB) cut-off. ${ }^{12}$

The combination of durvalumab either with tremelimumab and/or chemotherapy has been assessed in two Phase I clinical trials. ${ }^{31,32}$ The first phase I clinical trial enrolled 102 immunotherapy-naive metastatic NSCLC patients, reporting that the combination of durvalumab $20 \mathrm{mg} / \mathrm{kg}$ Q4W plus tremelimumab $1 \mathrm{mg} / \mathrm{kg}$ had a manageable tolerability profile, and anti-tumour activity irrespective of PDL1 status, being selected as the dose for Phase 3 studies. ${ }^{31}$ The second phase I clinical trial aimed to establish a recommended phase II dose for durvalumab with or without tremelimumab in combination with standard platinumdoublet chemotherapy. Out of 136 patients enrolled, 73 were NSCLC patients and the objective RR was $51 \%$ with a median PFS and OS of 6.5 months and 19.8 months, respectively. Anti-tumour activity was observed across PDL1 subtypes. The trial concluded that durvalumab at 1500 mg Q3W and tremelimumab $75 \mathrm{mg}$ Q3W can be safely combined with platinum-doublet chemotherapy. ${ }^{32}$ At present, there are three ongoing phase III clinical trials (MYSTIC, NEPTUNE and POSEIDON), which assess the efficacy of the combination of durvalumab plus tremelimumab in advanced NSCLC patients.

The randomized phase III MYSTIC trial assessed the efficacy of durvalumab (20 mg/kq Q4W) as monotherapy or in combination with tremelimumab $(1 \mathrm{mg} / \mathrm{kg}$ Q4W up to 4 doses) compared with platinum-based chemotherapy in 1118 treatment-naïve stage IV NSCLC patients. Primary endpoints were assessed in patients with PD-L1 expression $\geq 25 \%$ (by SP263 IHC, N=488). The combination of durvalumab plus tremelimumab did not meet the primary endpoint of improving PFS compared with platinumbased chemotherapy in patients with PD-L1 $\geq 25 \%$ (PFS: 3.9 vs 5.4 months, HR 1.05; 99.5\% CI, 0.722, 1.534; $\mathrm{p}=0.705$ ). Moreover, the results of two additional primary endpoints of OS benefit for durvalumab monotherapy (16.3 vs 12.9 months, HR 0.76 , 97.54\% CI $0.564-1.019$; nominal $\mathrm{p}=0.036$ ) and $\mathrm{OS}$ benefit for durvalumab plus tremelimumab (OS: 11.9 vs 12.9 months HR 0.85 , 98.77\% CI 0.611-1.173; nominal $\mathrm{p}=0.202$ ) compared with platinum-based chemotherapy were not statistically significant. ${ }^{33}$ Among patients who received subsequent treatment, ICI was administered to $10 / 73(13.7 \%)$ patients in the durvalumab arm and 64/95 (67.4\%) patients in the chemotherapy arm; most commonly nivolumab. An exploratory analysis reported that after adjusting for subsequent ICI treatment, durvalumab significantly improved OS compared to chemotherapy (HR, 0.66, 97.54\% CI: $0.49-0.90 ; p=0.002)$. However, although subsequent immunotherapy may have confounded the primary OS outcome, the control arm in the MYSTIC trial reflects the clinical daily approach, and data from an exploratory analysis may not endorse durvalumab monotherapy in the first line. ${ }^{34}$

Blood TMB (bTMB) has been reported as a predictive biomarker for survival benefit with anti- $\mathrm{PD}(\mathrm{L}) 1$ agents as monotherapy ${ }^{35,36}$ or in combination with chemotherapy. ${ }^{35}$ In the MYSTIC trial, a preplanned exploratory analysis examined survival according to bTMB, which could be determined in $72.4 \%$ of patients $(\mathrm{N}=809)$. For patients with a high bTMB ( $\geq 16 \mathrm{Mut} / \mathrm{Mb}, 39 \%$ of all patients in whom bTMB was assessed), the median OS were 16.5, 11.0 and 10.5 months, for durvalumab plus tremelimumab, 
durvalumab monotherapy and platinum-based chemotherapy, respectively. The corresponding 2-year OS rates were $39 \%, 30 \%$ and $18 \%$, respectively. The HR for OS for the combination compared with chemotherapy favoured the combination (HR 0.62; 95\% CI: 0.45-0.86), but durvalumab monotherapy did not improve the OS compared with chemotherapy (HR 0.80; 95\% CI: 0.59-1.07). ${ }^{33}$ Similarly, with a cut-off point of $b T M B \geq 20 \mathrm{Mut} / \mathrm{Mb}$, survival improvement was achieved with the combination of durvalumab plus tremelimumab compared with chemotherapy (21.9 months vs 10.0 months; HR 0.49 , 95\% CI: $0.32,0.74)$, but not with durvalumab compared with chemotherapy (12.6 months vs 10.0 months, HR $0.72,95 \%$ CI: $0.50,1.05){ }^{37}$

Specific gene mutations have been associated with resistance (STK11 and KEAPl) or sensitisation (ARIDIA) to anti-PD-(L)1 monotherapy. The assessment of these mutations in ctDNA were analysed in the MYSTIC trial. Among the mutation evaluable population $(\mathrm{N}=943)$, the incidence of mutations in STK11, KEAP1, and ARID1A were $16 \%$, $18 \%$ and $12 \%$, respectively, and STK11 and KEAPI were more prevalent in patients with non-squamous than squamous carcinoma. Shorter OS across all treatment arms were reported in patients with mutation in STK11 or KEAPI compared with STK11 or KEAP1 wild-type, whereas patients with $A R I D 1 A$ mutation had a longer median OS than patients with $A R I D I A$ wild-type in the ICI combo arm, but not in the durvalumab arm compared with chemotherapy. $^{38}$ These data support STK11 and KEAP1 as prognostic and $A R I D 1 A$ as predictive, but they are exploratory and require further validation.

The phase III NEPTUNE trial (NCT02542293) determined the efficacy of the combination of durvalumab plus tremelimumab versus platinum-based chemotherapy in first-line setting of stage IV NSCLC patients. On 6th June 2019, the primary endpoint of this study changed from all patients to OS in patients with $\mathrm{bTMB} \geq 20 \mathrm{Mut} /$ $\mathrm{Mb}$, and a press release on 21st August 2019 reported that the combination ICI arm did not meet the primary OS endpoint compared with chemotherapy. ${ }^{39}$ Therefore, the predictive biomarker role of TMB for the combination of durvalumab and tremelimumab remains a challenge in the absence of prospective validation for survival benefit.

Finally, the ongoing phase III POSEIDON trial (NCT03164616) evaluates durvalumab plus chemotherapy with or without tremelimumab or chemotherapy alone in the first-line setting in advanced NSCLC. Patients are not selected according to PD-L1 expression or high TMB. The co-primary endpoints are PFS according to independent review and OS. The estimated primary data completion is April 2021.

\section{Durvalumab with Targeted Therapies}

Although oncogenic addicted tumors may express PD-L1, in some cases even high, the benefit of ICI as monotherapy in these patients is scarce and available data support upfront targeted therapies in this population. ${ }^{40-43}$ Moreover, PD-L1 is often constitutively expressed in these patients and patients often have an immunesuppressed tumor micro-environment. ${ }^{44}$ The ATLANTIC trial evaluated durvalumab as third line or beyond in the $E G F R / A L K$ population, and reported a RR ranging from $12.2 \%$ to $30.9 \%$, PFS from 1.9 to 2.4 months, and 1-year OS from $40 \%$ to $51 \%$, based on the cut-off PD-L1 expression $\geq 25 \%$ or $\geq 90 \%$, but no responses were reported in the $A L K$-positive population. ${ }^{24}$

In the phase I TATTON trial, ${ }^{45}$ EGFR-mutant advanced NSCLC patients with disease progression on a prior EGFRtyrosine kinase inhibitor (TKI) were enrolled and allocated to dose-escalating cohorts combining osimertinib $80 \mathrm{mg}$ orally once a day with selumetinib (25-75 mg oral twice a day; continuous or intermittent), savolitinib (600-800 mg oral once a day), or durvalumab (3-10 $\mathrm{mg} / \mathrm{kg}$ intravenous Q2W). Among 23 patients enrolled in the durvalumab cohort, the RR was $44 \%$ with a median duration of response of 20.4 months. However, the combination of osimertinib with durvalumab was not feasible due to increased reporting of interstitial lung disease (ILD of grade $\geq 3$ of $10 \%$ and $7.7 \%$ at the dose of 3 and $10 \mathrm{mg} / \mathrm{kg}$, respectively). ${ }^{45}$ The phase III open label CAURAL trial (NCT02454933) ${ }^{46}$ investigated safety and efficacy of osimertinib $80 \mathrm{mg}$ qd plus durvalumab $10 \mathrm{mg} / \mathrm{kg}$ Q2W. While patient numbers were limited due to early recruitment termination as a result of the increased ILD incidence reported in TATTON, there was no clear benefit of combining osimertinib with durvalumab. One grade 2 ILD event was reported in the 12 patients who received the combination. The reason for the difference in reported ILD frequencies in the osimertinib plus durvalumab arm from the CAURAL and TATTON studies remains unclear. We can conclude that although preliminary phase I results suggest durvalumab plus gefitinib to be tolerable (NCT02088112), ${ }^{47}$ the benefit/risk ratio does not support the addition of anti-PD (L) 1 to TKI in daily clinical practice.

\section{Durvalumab in Stage III}

In locally advanced NSCLC patients, the randomized (2:1) phase III PACIFIC trial assessed the role of durvalumab 
(10 mg/kg Q2W) compared with placebo as consolidation treatment for 1 year in 713 patients without progression after concurrent chemo-radiotherapy. Of note, the last radiation dose was administered 1 to 42 days before randomization (2:1) and enrolment was not restricted to any threshold levels for PD-L1 expression. Availability of PD-L1 results was not necessary for inclusion. Randomization was stratified by patient age ( $<65$ years versus $\geq 65$ years), sex, and smoking history (current or former smoker versus never smoked). Although only half of patients completed 1 year of treatment, durvalumab achieved both co-primary endpoints with significant improvements versus placebo in the PFS (17.2 vs 5.6 months, HR $=0.51,95 \%$ CI: $0.41-63$, $\mathrm{p}<0.0001)$ and $\mathrm{OS}(\mathrm{HR}=0.68,95 \% \mathrm{CI}: 0.53-0.87$, $\mathrm{p}=0.00251) .{ }^{48,49}$ After a median follow-up of 33.3 months, the updated OS remained consistent with previously reported, with a reduction in the risk of death of $31 \%$. Median OS was not reached with durvalumab and was 29.1 months with placebo (stratified HR 0.69 [95\% CI: 0.55-0.86]). The 12-, 24- and 36-month OS rates with durvalumab and placebo were $83.1 \%$ versus $74.6 \%, 66.3 \%$ versus $55.3 \%$, and $57.3 \%$ versus $43.5 \%$, respectively. ${ }^{50}$ Only half of the patients received subsequent anticancer treatment at the time of progression in both arms $(43.3 \%$ and $57.8 \%$ of patients in durvalumab and placebo-arm, respectively); and in total $9.7 \%$ and $26.6 \%$, respectively, subsequently received immunotherapy (primarily, nivolumab or pembrolizumab). ${ }^{50}$ Durvalumab did also improve the secondary endpoints RR $30 \%$ vs $17.8 \%, \mathrm{p}<0.001$. Table 1$)^{48}$ and median time to death (30.2 vs 17.8 months, respectively, HR 0.61 ; 95\% CI,

Table I PACIFIC Trial

\begin{tabular}{|c|c|c|c|}
\hline & Durvalumab & Placebo & HR, p-value \\
\hline RR (\%) & 30 & 17.8 & $P<0.0001$ \\
\hline PFS (mo.) & 17.2 & 5.6 & HR $0.5 \mathrm{I}, \mathrm{p}<0.000 \mathrm{I}$ \\
\hline PD-LI $\geq 25 \%$ (mo.) & 17.8 & 3.7 & HR $0.4 \mathrm{I}(0.26-0.65)$ \\
\hline PD-LI < 25\% (mo.) & 16.9 & 6.9 & HR $0.59(0.43-0.82)$ \\
\hline PD-LI $\geq 1 \%$ (mo.) & 17.8 & 5.6 & HR $0.46(0.33-0.64)$ \\
\hline PD-LI < I\% (mo.) & 10.7 & 5.8 & HR $0.73(0.48-1.11)$ \\
\hline OS (mo.) & Not reached & 29.1 & HR $0.69(0.55-0.86)$ \\
\hline PD-LI $\geq 25 \%$ (mo.) & Not reached & 21.1 & HR $0.50(0.30-0.83)$ \\
\hline PD-LI < 25\% (mo.) & 39.7 & 37.7 & HR $0.89(0.63-1.25)$ \\
\hline PD-LI $\geq$ I\% (mo.) & Not reached & 29.1 & HR $0.53(0.36-0.77)$ \\
\hline PD-LI < I\% (mo.) & 33.1 & 45.6 & HR I.14 (0.7I-I.84) \\
\hline I-year OS (\%) & 83.1 & 74.1 & \\
\hline 2-year OS (\%) & 66.3 & 55.3 & \\
\hline 3-year OS (\%) & 57.3 & 43.5 & \\
\hline
\end{tabular}

Abbreviations: RR, response rate; PFS, progression-free survival; OS, overall survival; Mo, months; HR, hazard ratio.
0.49 to 0.75$).{ }^{50}$ Last, durvalumab decreased the incidence of new brain metastases (6.3\% vs $11.8 \%$, respectively). ${ }^{49}$ Of note, incidence of brain metastases was also in the comparator arm lower than in previously reported stage III NSCLC trials such as the NVALT11/DLCRG02 or RTOG 0214 $\left(\sim 27 \%\right.$ in the no prophylactic cranial irradiation arm) ${ }^{51,52}$ The reason for the low percentage of brain metastases in the placebo arm of the PACIFIC trial is unclear. Subgroup analyses showed a trend toward a greater PFS and OS benefit for patients randomized early after completion of radiotherapy ( $<14$ days). ${ }^{48-50}$ The safety profiles were similar between arms, with $30.5 \%$ vs $26.1 \%$ grade $\geq 3$ AEs, including pneumonitis (3.6\% vs 2.4\%); $15.4 \%$ durvalumab and $9.8 \%$ placebo treated patients discontinued the regimen due to AEs. ${ }^{48,49}$ Treatment exposure was similar in patients with or without pneumonitis across both arms as was median time to onset of pneumonitis from treatment start (55.0 days). Patients with pneumonitis were more likely to be Asian (47.9\% vs $17.6 \%)$ or with EGFR mutations (11.0\% vs 3.8\%). There were no apparent associations of pneumonitis with baseline respiratory disorders, prior radiotherapy dose, or prior cisplatin or carboplatin use. ${ }^{53}$ Of note, the occurrence of pneumonitis did not decrease the benefit of durvalumab. ${ }^{54}$ Finally, compared with placebo, the clinical benefit with durvalumab occurred without a detrimental effect on patient-reported outcomes, ${ }^{55}$ and consolidation strategy with durvalumab is cost-effective. ${ }^{56,57}$

The prespecified exploratory analysis regarding the impact of PD-L1 expression (by SP263 immunohistochemical assay) on the magnitude of durvalumab benefit was planned with a cut-off at $25 \%$ in tumor cells. However, only $64 \%$ of patients enrolled had tissue samples for testing PD-L1 expression, which were archival tissue samples before chemo-radiotherapy. Of these patients, $35 \%, 65 \%$, $67 \%, 33 \%$, and $32 \%$ had PD-L1 $\geq 25 \%,<25 \%, \geq 1 \%,<1 \%$, and $1-24 \%$, respectively. ${ }^{58}$ Durvalumab improved PFS compared with placebo in PD-L1 $\geq 25 \%$ (17.8 versus 3.7 months, HR 0.41 ; $95 \%$ CI: $0.26-0.65)$ and PD-L1 $<25 \%$ ( 16.9 versus 6.9 months; HR $0.59,95 \%$ CI: $0.43-0.82$ ), but only OS benefit was reported for patients with PD-L1 $\geq 25 \%$ (NR versus 21.1 months; HR: $0.50,95 \%$ CI: $0.30-0.83$ ) but not for PD-L1 <25\% (39.7 versus 37.4 months, HR: 0.89, 95\% CI: $0.63-1.25$. Table 1). ${ }^{58}$ However, at the request of EMA, an additional exploratory post hoc analysis was conducted with a PD-L1 expression-level cut-off of $1 \%$. In this post hoc analysis, 148 patients had PD-L1 $\leq 1 \%$ and durvalumab did not show PFS benefit (HR 0.73; 95\% CI: 0.48-1.11) compared with placebo, and a trend toward worse survival with 
durvalumab was reported in this subgroup (33.1 versus 45.6 months, HR: $1.14,95 \%$ CI: $0.71-1.84)^{58}$ (Table 1). While FDA approval of durvalumab in February 2018 was unrestricted, the EMA approval in September 2018 is limited to the PD-L1 $\geq 1 \%$ tumors, even if the statistical validity of this subgroup analysis is questionable and the negative impact should be prospectively tested. ${ }^{59}$

PACIFIC trial was the first trial demonstrating a survival improvement and manageable safety profile with ICI consolidation after concurrent chemoradiation in stage III NSCLC and established a new SoC, which has also been confirmed in real-life practice. ${ }^{60}$ The phase III PACIFIC5 trial (NCT03706690) will validate the results of durvalumab (1500 mg Q4W) compared with placebo after chemoradiation. Differences with the PACIFIC trial are the mandatory PD-L1 assessment before randomization, the allowance of sequential chemoradiation and the different treatment schedule (Q4W instead of Q2W, fixed dosing). Consolidation strategy with nivolumab is also being explored in the phase III RTOG 3505 trial (NCT02768558). ${ }^{61}$ However, several relevant clinical questions remain still open. It is important to address whether a similar benefit is observed with sequential chemoradiation therapy, assessed in phase II PACIFIC6 trial (NCT03693300), and the optimization of the potential synergy by administrating ICI concurrently with thoracic radiotherapy. The combination of ICI and chemoradiation has been tested in phase I clinical trial (NCT02621398) with pembrolizumab concurrent with chemoradiation reporting a promising 1-year PFS of $69.7 \%$ and median PFS of 18.9 months. $^{62}$ These results have been confirmed in the phase II LUN 14179 trial with concurrent pembrolizumab and chemoradiation (median and 1-year PFS of 17 months and $60.2 \%$, respectively, median OS not reached, and grade $\geq 3$ pneumonitis of $5.4 \%$ ). ${ }^{63}$ Other trials such as the DETERRED ${ }^{64}$ and NICOLAS trial ${ }^{65,66}$ are also assessing the concurrent strategy with atezolizumab and nivolumab, respectively. The DETERRED trial was conducted in two parts. In part $1(\mathrm{~N}=10)$ the chemoradiation was followed by consolidation chemotherapy (atezolizumab [two cycles] and maintenance atezolizumab up to 1 year). In part $2(\mathrm{~N}=30)$ patients received concurrent atezolizumab plus chemoradiation, followed by the same consolidation and maintenance therapies as in part 1 . The grade 3 AEs were $80 \%$, without differences in part 1 or 2 , and pneumonitis of grade 2 and above occurred in 10\%/ $16 \%$, respectively. The median PFS was 18.6 and 13.2 months, and OS was 22.8 months and not reached, in parts 1 and 2, respectively. ${ }^{64}$ The NICOLAS trial evaluates the safety and efficacy of nivolumab combined with chemoradiation in stage III NSCLC. The secondary endpoint of 1-year PFS rate was 54\%, with median PFS of 12.4 months and 1 -year OS of $79 \%$. No grade- $\geq 3$ pneumonitis was observed by the end of the 3-month post-RT followup period. ${ }^{65,66}$ Although these results endorse that concurrent ICI and chemoradiation is feasible and safe, the improvement in the outcome with this approach should be prospectively validated. This strategy is being ongoing tested in phase III PACIFIC2 trial (NCT03519971). The study was designed to assess the additional efficacy (PFS and RR) and safety of durvalumab when given concurrent with definitive chemoradiation. However, the control arm of PACIFIC2 trial is just chemoradiation alone. Whether concurrent ICI-chemoradiation followed by consolidation ICI improves outcome compared with consolidation ICI alone is unknown. Furthermore, several other trials are evaluating other immunotherapy strategies added to chemoradiation. For example, the phase II COAST trial (NCT03822351) evaluates durvalumab maintenance, versus durvalumab plus oleclumab (antiCD73) or plus monalizumab (antiNKG2Areceptor). The randomized phase II trial NCT03285321 evaluates nivolumab-ipilimumab consolidation. In an interim analysis $(\mathrm{N}=10)$, toxicity was manageable. ${ }^{67}$ The triple arm, randomized phase III Checkmate 73L (NCT04026412) trial is evaluating nivolumab concurrent with chemoradiation followed by nivolumab and ipilimumab maintenance, chemoradiation followed by durvalumab, and nivolumab concurrent with chemoradiation followed by nivolumab maintenance.

It is also important to select those patients who benefit the most of consolidation ICI strategy, as PD-L1 is an imperfect biomarker. Circulating tumor DNA (ctDNA) may detect minimal residual disease (MRD) following curative treatment-strategy. Recently, in a cohort of 65 patients receiving chemoradiation for stage III NSCLC, 28 receiving consolidation ICI, those with undetectable ctDNA (assessed by CAPPSeq) after chemoradiation had excellent outcome independently of consolidation ICI or not. In contrast, patients with MRD post-chemoradiation who received consolidation ICI had significantly better outcomes than patients who did not receive consolidation treatment. $^{68}$ Therefore, dynamic ctDNA evolution may help to personalise consolidation ICI strategy after CRT. However, prospective trials are necessary before such an approach be routinely applied in daily clinical practice. 


\section{$\mathrm{ICl}$ in the (Neo)-Adjuvant Setting, with} a Focus on Durvalumab

Considering the long-term benefit of ICI in the advanced setting, ${ }^{50,69}$ this strategy is being assessed in early-stage NSCLC. Although the presence of the antigens in the tumor may enhance the efficacy of ICI, and different preclinical studies suggested that a neoadjuvant ICI approach was associated with improved survival compared with the same adjuvant strategy, ${ }^{70,71}$ both strategies have been investigated in early-stage NSCLC patients.

For instance, several studies have reported the feasibility and safety of a neoadjuvant approach with anti-PD(L)1 agents as monotherapy, ${ }^{72-74}$ including durvalumab assessed in the IONESCO (NCT03030131) and NeoCOAST (NCT03794544) trial. Globally, monotherapy with anti-PD (L)- 1 reported a RR by RECIST criteria of $20 \%$ and almost $90 \%$ of patients underwent surgery with a pathological complete response (pCR) rate of $15 \% .^{72-74}$ In the neoadjuvant chemotherapy era, major pathological response (MPR), defined as $\leq 10 \%$ viable tumor cells after neoadjuvant treatment, was validated as surrogate of outcome. ${ }^{75,76}$ Although the correlation between MPR and long-term outcome with immunotherapy remains uncertain, clinical trials have adopted MPR as a potential endpoint. Of note, there is no correlation between RECIST and MPR, as reflected for the MPR rate ranging from $19 \%$ to $45 \%$ with neoadjuvant antiPD(L)1 in NSCLC. ${ }^{72-74}$ Globally, these data were very promising compared with the historical data with neoadjuvant chemotherapy (MPR $\sim 20 \%$ and $\mathrm{pCR} \leq 4 \%$ ). ${ }^{76}$ Other ongoing clinical trials are assessing anti-PD(L)-1 as neoadjuvant treatment such as PRINCEPS (NCT02994576), TOP-1501 (NCT02818920), CANOPY-N (NCT03968419).

Combination of ICI-ICI has also been evaluated in phase II NEOSTAR trial testing nivolumab plus ipilimumab or nivolumab monotherapy. ${ }^{77}$ The combination approach versus nivolumab monotherapy reported higher pCR (29\% versus 9\%) and MPR (33\% versus 17\%), but incidence of grade 3 immune-related adverse events (irAE's) was also higher (23\% versus $13 \%)$.

Likewise, neoadjuvant immune-chemotherapy combination has been also assessed in early-stage NSCLC in the NADIM (nivolumab plus chemotherapy in stage III) and in the trial by Shu et al (atezolizumab plus chemotherapy in stage IB-IIIA) patients who received atezolizumab plus chemotherapy. ${ }^{78,79}$ This strategy reported a meaningful clinical outcome in terms of MPR and pCR of $\sim 80 \%$ and $\sim 60 \%$, respectively, with $80 \%$ of patients receiving surgery.
These results endorse the neoadjuvant combination of anti$\mathrm{PD}(\mathrm{L}) 1$ plus chemotherapy approach as the most suitable to be tested in phase III clinical trials, such as KEYNOTE 617 with pembrolizumab (NCT03425643), CheckMate816 with nivolumab (NCT02998528), and IMpower030 with atezolizumab (NCT03456063), and the AEGEAN trial with durvalumab (NCT03800134). All except KEYNOTE 617 trials, the primary endpoint is MPR (OS for the pembrolizumab trial), and all include anti-PD(L)-1 monotherapy as adjuvant treatment for almost 1 year.

Contrary to resected melanoma where adjuvant ICI has become SoC, ${ }^{80-82}$ no clinical data on safety or efficacy with adjuvant ICI in resected early-stage NSCLC patients is available yet. However, several ongoing phase III clinical trials in resected stage IB-IIIA NSCLC assess adjuvant ICI for 1 year compared with placebo or observation after adjuvant chemotherapy, if indicated. The trials comparing adjuvant anti-PD(L)-1 with placebo are: BR31 trial (NCT02273375) with durvalumab, enrolling 1380 patients and PEARLS (NCT02504372) with pembrolizumab enrolling 1080 patients. The two trials comparing adjuvant antiPD(L)-1 with observation are the ANVIL trial (NCT02595944) with nivolumab, which enrols 903 patients and the IMpower130 trial (NCT02486718) with atezolizumab and enrolling 1280 patients. The primary endpoint for all these trials is disease-free survival (DFS), including DFS in PD-L1 positive tumors in BR31 trial and OS in ANVIL trial. Treatment compliance, lack of predictive biomarker, treatment duration, and DFS as primary endpoint instead of OS are potential concerns of these trials. Table 2 summarizes the ongoing clinical trials with durvalumab in different stages.

Table 2 Ongoing Clinical Trials with Durvalumab in Non-Small Cell Lung Cancer Patients

\begin{tabular}{|l|l|l|}
\hline Trial Name & NCT Number & Setting \\
\hline PEARL & NCT03003962 & Metastatic \\
LUNG MATRIX TRIAL & NCT02664935 & Metastatic \\
NEPTUNE & NCT02542293 & Metastatic \\
POSEIDON & NCT031646I6 & Metastatic \\
PACIFIC2 & NCT035I997I & Locally advanced \\
PACIFIC5 & NCT03706690 & Locally advanced \\
COAST & NCT0382235I & Locally advanced \\
BR3I & NCT02273375 & Adjuvant \\
IONESCO & NCT03030I3I & Neoadjuvant \\
NeoCOAST & NCT03794544 & Neoadjuvant \\
AEGEAN & NCT03800I34 & Neoadjuvant \\
\hline
\end{tabular}




\section{Rechallenge}

Nowadays, ICIs are SoC in metastatic and locally advanced disease (when treated with concurrent chemoradiation), and in the coming future, they will probably be also $\mathrm{SoC}$ in the (neo)-adjuvant setting. Therefore, the potential benefit of rechallenge strategy for those patients who become metastatic and who have already received ICI in the early stages remains a relevant clinical challenge. The limited evidence about the rechallenge strategy in NSCLC comes from the metastatic setting. Durvalumab activity in 70 previously treated patients across 14 different tumor types who stopped durvalumab after 1 year without disease progression was assessed. Rechallenge with durvalumab reported a RR of $11.4 \%$, with a diseases control rate (DCR) of $71.4 \%$. Median duration of response was 16.5 months. DCR at $\geq 24$ weeks was $47.1 \%$ and PFS at 12 months was $34.2 \%$, with similar rates observed regardless of tumor type, suggesting the efficacy of rechallenge. However, progressive disease rate was $23 \%$ at rechallenge compared with $9 \%$ at the induction phase. ${ }^{83}$ However, both the heterogeneity in the cancer population enrolled and the limited number of patients do not aim to obtain firm conclusions. Similarly, data coming from the KEYNOTE 010 trial $^{13}$ and the KEYNOTE 024 trial, $^{29}$ retreatment with pembrolizumab at the time of progression after completing the number of cycles per protocol reported a disease control rate (DCR) of 70\%. However, this strategy was assessed in only 24 patients, and rechallenge mainly benefits those patients with an elapsed time between last dose of pembrolizumab and rechallenge of at least 1 year. ${ }^{13,29}$ Likewise, in daily clinical cohorts, rechallenge benefits those patients with a longer treatment duration with initial ICI treatment ${ }^{84}$ and those not receiving chemotherapy between first ICI treatment and rechallenge. ${ }^{85}$ However, the CheckMate 153 trial reported no benefit with nivolumab rechallenge in 39 patients who progressed during the surveillance period after completing 1-year of nivolumab treatment. ${ }^{86}$ Despite that there exists some evidence about the potential clinical benefit with rechallenge, it remains unknown what are the most suitable patients for this strategy, what is the optimal time-interval after finishing ICI in early stages for receiving ICI at the time of progression, and whether rechallenge is the most suitable strategy for those patients who stopped the ICI in early stages due to ir-AE's.

\section{Conclusions}

So far, durvalumab has proven its added value in improving PFS and OS, with maintaining QoL in stage III
NSCLC patients treated with concurrent chemoradiation. The place of durvalumab in the metastatic setting is challenging with the abundance of other ICIs that already have been improved either as monotherapy or in combination with chemotherapy. Durvalumab is not approved in this setting, and it is currently not possible to identify the metastatic NSCLC patient population that will have benefits of durvalumab, although also for durvalumab, higher PD-L1 expression is associated with more benefit. In early-stage NSCLC, the role of durvalumab as (neo)adjuvant treatment is being tested in different ongoing phase III clinical trials. The challenge for the future will be to find biomarkers in all disease stages that are predictive for ICI benefit (not only durvalumab benefit), and to define the optimal treatment strategy for each patient.

\section{Disclosure}

SG reports no conflicts of intertest related to the current work or outside this one.

JR reports no conflicts of interest related to the current work; is a member of advisory board in Boehringer, BMS, and OSE-Immunotherapeutics; and speaker for Pfizer; received travel fees from OSE-immunotherapeutics, BMS, AstraZeneca, and Roche outside the current work.

LH reports no conflicts of interest related to the current work; received research funding from Roche, Boehringer Ingelheim, AstraZeneca (all institution); and is a member of advisory board in Boehringer, BMS, Lilly, Roche, Pfizer, Takeda, MSD, Boehringer Ingelheim (all institution); speaker for MSD; received travel/conference reimbursement from Roche, BMS (self); grants for mentorship program with key opinion leaders funded by AstraZeneca; fees for educational webinars from Quadia (self), and fees for interview sessions funded by Roche (institution) outside of current work.

GL reports no conflicts of interest related to the current work; received institutions grants from Merck, Roche Genentech, AstraZeneca, BMS, EMD Serono, Novartis, Tesaro, Blueprint medicines, G1 Therapeutics US NIH/ NCI, Singapore A-Star, Brazil CNPq outside the current work. The authors report no other conflicts of interest in this work.

\section{References}

1. Reck M, Rodríguez-Abreu D, Robinson AG, et al. Pembrolizumab versus chemotherapy for PD-L1-positive non-small-cell lung cancer. $N$ Engl J Med. 2016;375(19):1823-1833. doi:10.1056/NEJMoa1606 774 
2. Mok TSK, Wu Y-L, Kudaba I, et al. Pembrolizumab versus chemotherapy for previously untreated, PD-L1-expressing, locally advanced or metastatic non-small-cell lung cancer (KEYNOTE-042): a randomised, open-label, controlled, phase 3 trial. Lancet. 2019;393 (10183):1819-1830. doi:10.1016/S0140-6736(18)32409-7

3. Gandhi L, Rodríguez-Abreu D, Gadgeel S, et al. Pembrolizumab plus chemotherapy in metastatic non-small-cell lung cancer. $N$ Engl $J$ Med. 2018;378(22):2078-2092. doi:10.1056/NEJMoa1801005

4. Gadgeel SM, Garassino MC, Esteban E, et al. KEYNOTE-189: updated OS and progression after the next line of therapy (PFS2) with pembrolizumab (pembro) plus chemo with pemetrexed and platinum vs placebo plus chemo for metastatic nonsquamous NSCLC. JCO. 2019;37 (15_suppl):9013. doi:10.1200/JCO.2019.37.15_suppl.9013

5. West H, McCleod M, Hussein M, et al. Atezolizumab in combination with carboplatin plus nab-paclitaxel chemotherapy compared with chemotherapy alone as first-line treatment for metastatic non-squamous non-small-cell lung cancer (IMpower130): a multicentre, randomised, open-label, phase 3 trial. Lancet Oncol. 2019;20(7):924-937. doi:10.1016/S1470-2045(19)30167-6

6. Socinski MA, Jotte RM, Cappuzzo F, et al. Atezolizumab for first-line treatment of metastatic nonsquamous NSCLC. $N$ Engl $J$ Med. 2018;378(24):2288-2301. doi:10.1056/NEJMoa1716948

7. Socinski M, Jotte RM, Cappuzzo F, et al. Overall survival analysis of IMpower150, a randomized $\mathrm{Ph} 3$ study of atezolizumab + chemotherapy \pm bevacizumab vs chemo + bev in $1 \mathrm{~L}$ nonsquamous NSCLC. J Clin Oncol. 2018;36(15_suppl):9002. doi:10.1200/JCO.2018.36. 15 suppl.9002

8. Paz-Ares L, Luft A, Vicente D, et al. Pembrolizumab plus chemotherapy for squamous non-small-cell lung cancer. $N$ Engl J Med. 2018;379(21):2040-2051. doi:10.1056/NEJMoa1810865

9. Brahmer JR, Rodríguez-Abreu D, Robinson AG, et al. Health-related quality-of-life results for pembrolizumab versus chemotherapy in advanced, PD-L1-positive NSCLC (KEYNOTE-024): a multicentre, international, randomised, open-label phase 3 trial. Lancet Oncol. 2017;18(12):1600-1609. doi:10.1016/S1470-2045(17)30690-3

10. Garassino MC, Gadgeel S, Esteban E, et al. Patient-reported outcomes following pembrolizumab or placebo plus pemetrexed and platinum in patients with previously untreated, metastatic, non-squamous non-small-cell lung cancer (KEYNOTE-189): a multicentre, double-blind, randomised, placebo-controlled, phase 3 trial. Lancet Oncol. 2020;21(3):387-397. doi:10.1016/S14702045(19)30801-0

11. Mazieres J, Kowalski D, Luft A, et al. Health-related quality of life with carboplatin-paclitaxel or nab-paclitaxel with or without pembrolizumab in patients with metastatic squamous non-small-cell lung cancer. J Clin Oncol. 2020;38(3):271-280. doi:10.1200/JCO.19. 01348

12. Hellmann MD, Paz-Ares L, Bernabe Caro R, et al. Nivolumab plus ipilimumab in advanced non-small-cell lung cancer. $N$ Engl J Med. 2019;381(21):2020-2031. doi:10.1056/NEJMoa1910231

13. Herbst RS, Garon EB, Kim D-W, et al. Long-term outcomes and retreatment among patients with previously treated, programmed death-ligand 1-positive, advanced non-small-cell lung cancer in the KEYNOTE-010 study. J Clin Oncol. 2020:JCO1902446. doi:10.12 00/JCO.19.02446

14. Borghaei H, Paz-Ares L, Horn L, et al. Nivolumab versus docetaxel in advanced nonsquamous non-small-cell lung cancer. $N$ Engl J Med. 2015;373(17):1627-1639. doi:10.1056/NEJMoa1507643

15. Brahmer J, Reckamp KL, Baas P, et al. Nivolumab versus docetaxel in advanced squamous-cell non-small-cell lung cancer. $N$ Engl J Med. 2015;373(2):123-135. doi:10.1056/NEJMoa1504627

16. Fehrenbacher L, von Pawel J, Park K, et al. Updated efficacy analysis including secondary population results for oak: a randomized phase iii study of atezolizumab versus docetaxel in patients with previously treated advanced non-small cell lung cancer. J Thorac Oncol. 2018;13(8):1156-1170. doi:10.1016/j.jtho.2018.04.039
17. Garon EB, Hellmann MD, Rizvi NA, et al. Five-year overall survival for patients with advanced non-small-cell lung cancer treated with pembrolizumab: results from the phase I KEYNOTE-001 study. J Clin Oncol. 2019;37(28):2518-2527. doi:10.1200/JCO.19.00934

18. Gettinger S, Horn L, Jackman D, et al. Five-year follow-up of nivolumab in previously treated advanced non-small-cell lung cancer: results from the CA209-003 study. J Clin Oncol. 2018;36 (17):1675-1684. doi:10.1200/JCO.2017.77.0412

19. Gettinger S, Borghaei H, Brahmer J, et al. Five-year outcomes from the randomized, phase 3 trials checkMate 017/057: nivolumab vs Docetaxel in Previously Treated NSCLC. J Thoracic Oncol. 2019;14(10):S244-S245. doi:10.1016/j.jtho.2019.08.486

20. Gadgeel S, Rodríguez-Abreu D, Speranza G, et al. Updated analysis from KEYNOTE-189: pembrolizumab or placebo plus pemetrexed and platinum for previously untreated metastatic nonsquamous non-small-cell lung cancer. J Clin Oncol. 2020:JCO1903136. doi:10.1200/JCO.19.03136

21. Stewart R, Morrow M, Hammond SA, et al. Identification and characterization of MEDI4736, an Antagonistic Anti-PD-L1 monoclonal antibody. Cancer Immunol Res. 2015;3(9):1052. doi:10.1158/23266066.CIR-14-0191

22. Murakami S. Durvalumab for the treatment of non-small cell lung cancer. Expert Rev Anticancer Ther. 2019;19(12):1009-1016. doi:10.1080/14737140.2019.1699407

23. Antonia SJ, Balmanoukian A, Brahmer J, et al. Clinical activity, tolerability, and long-term follow-up of durvalumab in patients with advanced NSCLC. J Thorac Oncol. 2019;14(10):1794-1806. doi:10.1016/j.jtho.2019.06.010

24. Garassino MC, Cho B-C, Kim J-H, et al. Durvalumab as third-line or later treatment for advanced non-small-cell lung cancer (ATLANTIC): an open-label, single-arm, Phase 2 study. Lancet Oncol. 2018;19(4):521-536. doi:10.1016/S1470-2045(18)30144-X

25. Planchard D, Reinmuth N, Orlov S, et al. ARCTIC: durvalumab with or without tremelimumab as third-line or later treatment of metastatic non-small-cell lung cancer. Ann Oncol. 2020;31(5):609-618. doi:10.1016/j.annonc.2020.02.006

26. Papadimitrakopoulou V, Redman MW, Borghaei H, et al. Phase II study of durvalumab (MEDI4736) for previously treated patients with stage IV squamous NSCLC (SqNSCLC): lung-MAP Sub-study SWOG S1400A. Ann Oncol. 2017;28(suppl_2):ii29. doi:10.1093/annonc/mdx091.003

27. Planchard D, Popat S, Kerr K, et al. Metastatic non-small cell lung cancer: ESMO Clinical Practice Guidelines for diagnosis, treatment and follow-up. Ann Oncol. 2018;29(Supplement_4):iv192-iv237. doi:10.1093/annonc/mdy275

28. Aguilar EJ, Ricciuti B, Gainor JF, et al. Outcomes to first-line pembrolizumab in patients with non-small-cell lung cancer and very high PD-L1 expression. Ann Oncol. 2019;30(10):1653-1659. doi:10.1093/annonc/mdz288

29. Reck M, Rodríguez-Abreu D, Robinson AG, et al. KEYNOTE-024 3-year survival update: pembrolizumab vs platinum-based chemotherapy for advanced non-small-cell lung cancer. $J$ Thoracic Oncol. 2019;14(10):S243. doi:10.1016/j.jtho.2019.08.483

30. Spigel D, de Marinis F, Giaccone G, et al. IMpower110: interim overall survival (OS) analysis of a phase III study of atezolizumab (atezo) vs platinum-based chemotherapy (chemo) as first-line (1L) treatment (tx) in PD-L1-selected NSCLC. Ann Oncol. 2019;30 (mdz293):v915. doi:10.1093/annonc/mdz293

31. Antonia S, Goldberg SB, Balmanoukian A, et al. Safety and antitumour activity of durvalumab plus tremelimumab in non-small cell lung cancer: a multicentre, phase 1b study. Lancet Oncol. 2016;17 (3):299-308. doi:10.1016/S1470-2045(15)00544-6

32. Juergens RA, Hao D, Ellis PM, et al. A phase IB study of durvalumab with or without tremelimumab and platinum-doublet chemotherapy in advanced solid tumours: canadian Cancer Trials Group Study IND226. Lung Cancer. 2020;143:1-11. doi:10.1016/j.lungcan.2020. 02.016 
33. Rizvi NA, Chul Cho B, Reinmuth N, et al. Durvalumab with or without tremelimumab vs platinum-based chemotherapy as first-line treatment for metastatic non-small cell lung cancer: MYSTIC. Ann Oncol. 2018;29(suppl_10):x40-x41. doi:10.1093/annonc/mdy511.005

34. Reinmuth N, Cho BC, Lee $\mathrm{KH}$, et al. Effect of post-study immunotherapy (IO) on overall survival (OS) outcome in patients with metastatic (m) NSCLC treated with first-line durvalumab (D) vs chemotherapy (CT) in the phase III MYSTIC study. Ann Oncol. 2019;30:ii77. doi:10.1093/annonc/mdz094.003

35. Aggarwal C, Thompson JC, Chien AL, et al. Baseline plasma tumor mutation burden predicts response to pembrolizumab-based therapy in patients with metastatic non-small cell lung cancer. Clin Cancer Res. 2020;26(10):2354-2361. doi:10.1158/1078-0432.CCR-19-3663

36. Gandara DR, Paul SM, Kowanetz M, et al. Blood-based tumor mutational burden as a predictor of clinical benefit in non-small-cell lung cancer patients treated with atezolizumab. Nat Med. 2018;24 (9):1441-1448. doi:10.1038/s41591-018-0134-3

37. Peters S, Cho BC, Reinmuth N, et al. Tumor mutational burden (TMB) as a biomarker of survival in metastatic non-small cell lung cancer (mNSCLC): blood and tissue TMB analysis from MYSTIC, a Phase III study of first-line durvalumab \pm tremelimumab vs chemotherapy. Cancer Res. 2019;79(13 Supplement):CT074. doi:10.11 58/1538-7445.AM2019-CT074

38. Rizvi N, Cho BC, Reinmuth N, et al. Mutations associated with sensitivity or resistance to immunotherapy in mNSCLC: analysis from the MYSTIC Trial. J Thoracic Oncol. 2019;14(10):S217. doi:10.1016/j.jtho.2019.08.428

39. Update on the Phase III NEPTUNE trial of Imfinzi plus tremelimumab in Stage IV non-small cell lung cancer; 2019. Available from: https://www.astrazeneca.com/media-centre/press-releases/2019/ update-on-the-phase-iii-neptune-trial-of-imfinzi-plus-tremelimumabin-stage-iv-non-small-cell-lung-cancer-21082019.html.

40. Mazieres J, Drilon A, Lusque A, et al. Immune checkpoint inhibitors for patients with advanced lung cancer and oncogenic driver alterations: results from the IMMUNOTARGET registry. Ann Oncol. 2019;30(8):1321-1328. doi:10.1093/annonc/mdz167

41. Sabari JK, Leonardi GC, Shu CA, et al. PD-L1 expression, tumor mutational burden, and response to immunotherapy in patients with MET exon 14 altered lung cancers. Ann Oncol. 2018;29 (10):2085-2091. doi:10.1093/annonc/mdy334

42. Offin M, Guo R, Wu SL, et al. Immunophenotype and response to immunotherapy of RET-rearranged lung cancers. JCO Precis Oncol. 2019:3. doi:10.1200/PO.18.00386

43. Dudnik E, Peled N, Nechushtan H, et al. BRAF mutant Lung Cancer: Programmed Death Ligand 1 Expression, Tumor Mutational Burden, Microsatellite Instability Status, And Response To Immune Check-Point Inhibitors. J Thorac Oncol. 2018;13(8):1128-1137. doi:10.1016/j.jtho.2018.04.024

44. Toki MI, Mani N, Smithy JW, et al. Immune marker profiling and programmed death ligand 1 expression across NSCLC mutations. $J$ Thorac Oncol. 2018;13(12):1884-1896. doi:10.1016/j.jtho.2018.09.012

45. Oxnard GR, Yang JC-H, Yu H, et al. TATTON: a multi-arm, phase Ib trial of osimertinib combined with selumetinib, savolitinib, or durvalumab in EGFR-mutant lung cancer. Ann Oncol. 2020;31 (4):507-516. doi:10.1016/j.annonc.2020.01.013

46. Yang JC-H, Shepherd FA, Kim D-W, et al. Osimertinib plus durvalumab versus osimertinib monotherapy in EGFR T790M-positive NSCLC following previous EGFR TKI therapy: CAURAL brief report. J Thorac Oncol. 2019;14(5):933-939. doi:10.1016/j.jtho.20 19.02 .001

47. Gibbons DL, Chow LQ, Kim D-W, et al. Efficacy, safety and tolerability of MEDI4736 (durvalumab [D]), a human IgG1 antiprogrammed cell death-ligand-1 (PD-L1) antibody, combined with gefitinib (G): A phase I expansion in TKI-naïve patients (pts) with EGFR mutant NSCLC. J Thoracic Oncol. 2016;11(4):S79. doi:10. 1016/S1556-0864(16)30171-X
48. Antonia SJ, Villegas A, Daniel D, et al. Durvalumab after chemoradiotherapy in stage III Non-small-cell lung cancer. $N$ Engl J Med. 2017;377(20):1919-1929. doi:10.1056/NEJMoa1709937

49. Antonia SJ, Villegas A, Daniel D, et al. Overall survival with durvalumab after chemoradiotherapy in stage III NSCLC. $N$ Engl J Med. 2018;379(24):2342-2350. doi:10.1056/NEJMoa1809697

50. Gray JE, Villegas A, Daniel D, et al. Three-year overall survival with durvalumab after chemoradiotherapy in stage III NSCLC-update from PACIFIC. J Thorac Oncol. 2020;15(2):288-293. doi:10.1016/ j.jtho.2019.10.002

51. De Ruysscher D, Dingemans A-MC, Praag J, et al. Prophylactic cranial irradiation versus observation in radically treated stage iii non-small-cell lung cancer: a randomized phase III NVALT-11/ DLCRG-02 Study. JCO. 2018;36(23):2366-2377. doi:10.1200/ JCO.2017.77.5817

52. Sun A, Hu C, Wong SJ, et al. Prophylactic cranial irradiation vs observation in patients with locally advanced non-small cell lung cancer: a long-term update of the NRG Oncology/RTOG 0214 Phase 3 randomized clinical trial. JAMA Oncol. 2019;5(6):847-855. doi:10.1001/jamaoncol.2018.7220

53. Vansteenkiste J, Naidoo J, Faivre-Finn C, et al. PACIFIC subgroup analysis: pneumonitis in stage III, unresectable NSCLC patients treated with durvalumab vs. placebo after CRT. J Thoracic Oncol. 2018;13(10):S370-S371. doi:10.1016/j.jtho.2018.08.350

54. Vansteenkiste JF, Naidoo J, Faivre-Finn C, et al. Efficacy of durvalumab in patients with stage III NSCLC who experience pneumonitis (PACIFIC). Ann Oncol. 2019;30:v592-v593. doi:10.1093/annonc/ mdz259.002

55. Hui R, Özgüroğlu M, Villegas A, et al. Patient-reported outcomes with durvalumab after chemoradiotherapy in stage III, unresectable non-small-cell lung cancer (PACIFIC): a randomised, controlled, phase 3 study. Lancet Oncol. 2019;20(12):1670-1680. doi:10.1016/ S1470-2045(19)30519-4

56. Panje CM, Lupatsch JE, Barbier M, et al. A cost-effectiveness analysis of consolidation immunotherapy with durvalumab in stage III NSCLC responding to definitive radiochemotherapy in Switzerland. Ann Oncol. 2020;31(4):501-506. doi:10.1016/j.annonc.2020.01.007

57. Criss SD, Mooradian MJ, Sheehan DF, et al. Cost-effectiveness and budgetary consequence analysis of durvalumab consolidation therapy vs no consolidation therapy after chemoradiotherapy in stage iii non-small cell lung cancer in the context of the US health care system. JAMA Oncol. 2019;5(3):358-365. doi:10.1001/jamaoncol.20 18.5449

58. Paz-Ares L, Spira A, Raben D, et al. Outcomes with durvalumab by tumour PD-L1 expression in unresectable, Stage III non-small-cell lung cancer in the PACIFIC trial. Ann Oncol. 2020;31(6):798-806. doi:10.1016/j.annonc.2020.03.287

59. Peters S, Dafni U, Boyer M, et al. Position of a panel of international lung cancer experts on the approval decision for use of durvalumab in stage III non-small-cell lung cancer (NSCLC) by the Committee for Medicinal Products for Human Use (CHMP). Ann Oncol. 2019;30 (2):161-165. doi:10.1093/annonc/mdy553

60. Girard N, Mornex F, Christoph DC, et al. PACIFIC-R: first real-world study of patients with unresectable, stage III NSCLC treated with durvalumab after chemoradiotherapy. Ann Oncol. 2019;30(Suppl 2): ii37. doi:10.1093/annonc/mdz067.017

61. Gerber DE, Urbanic JJ, Langer C, et al. Treatment Design and rationale for a randomized trial of cisplatin and etoposide plus thoracic radiotherapy followed by nivolumab or placebo for locally advanced non-small-cell lung cancer (RTOG 3505). Clin Lung Cancer. 2017;18(3):333-339. doi:10.1016/j.cllc.2016.10.009

62. Jabbour SK, Berman AT, Decker RH, et al. Prospective phase I multi-institutional trial of PD-1 blockade with pembrolizumab during concurrent chemoradiation for locally advanced, unresectable non-small cell lung cancer. JCO. 2019;37(15_suppl):8511. doi:10.12 00/JCO.2019.37.15_suppl.8511 
63. Durm G, Althouse S, Sadiq A, et al. Updated results of a phase II trial of concurrent chemoradiation with consolidation pembrolizumab in patients with unresectable stage III NSCLC. J Thoracic Oncol. 2018;13(10):S321. doi:10.1016/j.jtho.2018.08.238

64. Lin SH, Lin Y, Yao L, et al. Phase II trial of concurrent atezolizumab with chemoradiation for unresectable NSCLC. J Thorac Oncol. 2020;15(2):248-257. doi:10.1016/j.jtho.2019.10.024

65. Peters S, Felip E, Dafni U, et al. Safety evaluation of nivolumab added concurrently to radiotherapy in a standard first line chemo-radiotherapy regimen in stage III non-small cell lung cancer-The ETOP NICOLAS trial. Lung Cancer. 2019;133:83-87. doi:10.1016/j.lungcan.2019.05.001

66. Peters S, Felip E, Dafni U, et al. Efficacy evaluation of concurrent nivolumab addition to a first-line, concurrent chemo-radiotherapy regimen in unresectable locally advanced NSCLC: results from the European Thoracic Oncology Platform (ETOP 6-14) NICOLAS phase II trial. Ann Oncol. 2019;30:v591. doi:10.1093/annonc/mdz259

67. Yan M, Durm GA, Mamdani H, et al. Interim safety analysis of consolidation nivolumab and ipilimumab versus nivolumab alone following concurrent chemoradiation for unresectable stage IIIA/ IIIB NSCLC: big Ten Cancer Research Consortium LUN 16-081. JCO. 2019;37(15_suppl):8535. doi:10.1200/JCO.2019.37.15_suppl. 8535

68. Moding EJ, Liu Y, Nabet BY, et al. Circulating tumor DNA dynamics predict benefit from consolidation immunotherapy in locally advanced non-small-cell lung cancer. Nat Cancer. 2020;1(2): 176-183. doi:10.1038/s43018-019-0011-0

69. Topalian SL, Hodi FS, Brahmer JR, et al. Five-year survival and correlates among patients with advanced melanoma, renal cell carcinoma, or non-small cell lung cancer treated with nivolumab. JAMA Oncol. 2019;5(10):1411. doi:10.1001/jamaoncol.2019.2187

70. Liu J, Blake SJ, Yong MCR, et al. Improved efficacy of neoadjuvant compared to adjuvant immunotherapy to eradicate metastatic disease. Cancer Discov. 2016;6(12):1382-1399. doi:10.1158/2159-8290.CD$16-0577$

71. Cascone T, Hamdi H, Zhang F, et al. Superior efficacy of neoadjuvant compared to adjuvant immune checkpoint blockade in non-small cell lung cancer. Cancer Res. 2018;78(13 Supplement):1719. doi:10.1158/1538-7445.AM2018-1719

72. Forde PM, Chaft JE, Smith KN, et al. Neoadjuvant PD-1 blockade in resectable lung cancer. $N$ Engl J Med. 2018;378(21):1976-1986. doi:10.1056/NEJMoa1716078

73. Bar J, Urban D, Ofek E, et al. Neoadjuvant pembrolizumab (Pembro) for early stage non-small cell lung cancer (NSCLC): updated report of a phase I study, MK3475-223. JCO. 2019;37(15_suppl):8534. doi:10.1200/JCO.2019.37.15_suppl.8534

74. Gao S, Li N, S G, et al. Neoadjuvant PD-1 inhibitor (Sintilimab) in non-small cell lung cancer. J Thorac Oncol. 2020;15(5):816-826. doi: $10.1016 /$ j.jtho.2020.01.017
75. Betticher DC, Hsu Schmitz S-F, Tötsch M, et al. Prognostic factors affecting long-term outcomes in patients with resected stage IIIA pN2 non-small-cell lung cancer: 5-year follow-up of a phase II study. $\mathrm{Br}$ $J$ Cancer. 2006;94(8):1099-1106. doi:10.1038/sj.bjc.6603075

76. Hellmann MD, Chaft JE, William WN, et al. Pathological response after neoadjuvant chemotherapy in resectable non-small-cell lung cancers: proposal for the use of major pathological response as a surrogate endpoint. Lancet Oncol. 2014;15(1):e42-e50. doi:10. 1016/S1470-2045(13)70334-6

77. Cascone T, William WN, Weissferdt A, et al. Neoadjuvant nivolumab (N) or nivolumab plus ipilimumab (NI) for resectable non-small cell lung cancer (NSCLC): clinical and correlative results from the NEOSTAR study. JCO. 2019;37(15_suppl):8504. doi:10.1200/ JCO.2019.37.15_suppl.8504

78. Provencio M, Nadal E, Insa A, et al. NADIM study: updated clinical research and outcomes. J Thoracic Oncol. 2019;14(10):S241. doi:10.1016/j.jtho.2019.08.480

79. Shu CA, Grigg C, Chiuzan C, et al. Neoadjuvant atezolizumab + chemotherapy in resectable non-small cell lung cancer (NSCLC). JCO. 2018;36(15_suppl):8532. doi:10.1200/JCO.2018.36.15_suppl. 8532

80. Eggermont AMM, Chiarion-Sileni V, Grob -J-J, et al. Prolonged survival in stage III melanoma with ipilimumab adjuvant therapy. $N$ Engl J Med. 2016;375(19):1845-1855. doi:10.1056/NEJMoa161 1299

81. Weber JS, Del Vecchio M, Mandala M, et al. Adjuvant nivolumab (NIVO) versus ipilimumab (IPI) in resected stage III/IV melanoma: 3-year efficacy and biomarker results from the phase III CheckMate 238 trial. Ann Oncol. 2019;30(mdz255):v533-v534. doi:10.1093/ annonc/mdz255

82. Eggermont AMM, Blank CU, Mandala M, et al. Adjuvant pembrolizumab versus placebo in resected stage III melanoma. $N$ Engl J Med. 2018;378(19):1789-1801. doi:10.1056/NEJMoa1802357

83. Sheth S, Gao C, Mueller N, Martinez P, Soria J-C. Durvalumab activity in previously treated patients who stopped durvalumab without disease progression. Ann Oncol. 2019;30(mdz253.001):v475v476. doi:10.1093/annonc/mdz253.001

84. Giaj Levra M, Cotté F-E, Corre R, et al. Immunotherapy rechallenge after nivolumab treatment in advanced non-small cell lung cancer in the real-world setting: A national data base analysis. Lung Cancer. 2019;140:99-106. doi:10.1016/j.lungcan.2019.12.017

85. Gobbini E, Toffart AC, Perol M, et al. Immune checkpoint inhibitor (ICPi) re-challenge: outcomes analysis in a french national cohort of non-small-cell lung cancer (NSCLC) Patients. J Thoracic Oncol. 2019;14(10):S274. doi:10.1016/j.jtho.2019.08.550

86. Spigel DR, McLeod M, Hussein MA, et al. Randomized results of fixed-duration (1-yr) vs continuous nivolumab in patients (pts) with advanced non-small cell lung cancer (NSCLC). Ann Oncol. 2017;28 (suppl_5):v461. doi:10.1093/annonc/mdx380.002
OncoTargets and Therapy

\section{Publish your work in this journal}

OncoTargets and Therapy is an international, peer-reviewed, open access journal focusing on the pathological basis of all cancers, potential targets for therapy and treatment protocols employed to improve the management of cancer patients. The journal also focuses on the impact of management programs and new therapeutic agents and protocols on patient perspectives such as quality of life, adherence and satisfaction. The manuscript management system is completely online and includes a very quick and fair peer-review system, which is all easy to use. Visit http://www.dovepress.com/ testimonials.php to read real quotes from published authors. 